\title{
Iron intake and body iron stores, anaemia and risk of hyperglycaemia among Chinese adults: the prospective Jiangsu Nutrition Study (JIN)
}

\author{
Zumin Shi ${ }^{1,2, *}$, Minghao Zhou ${ }^{1}$, Baojun Yuan ${ }^{1}$, Lu ${ }^{3}{ }^{3}$, Yue Dai ${ }^{1}$, Yazhou Luo ${ }^{1}$ and \\ Gerd Holmboe-Ottesen ${ }^{2}$ \\ 'Jiangsu Provincial Center for Disease Control and Prevention, 172 Jiangsu Road, Nanjing 210009, People's \\ Republlc of China: ${ }^{2}$ Institute of General Practice and Community Medicine, University of Oslo, Oslo, Norway: \\ ${ }^{3}$ Department of Nutrition and Epidemiology, Harvard School of Public Health, Boston, MA, USA
}

Submitted 21 May 2009: Accepted 23 September 2009: First published online 28 October 2009

\begin{abstract}
Objective: Fe supplementation has been used to prevent anaemia in China; however, high $\mathrm{Fe}$ intake and body Fe stores may increase diabetes risk. The present study aimed to prospectively examine the association between Fe intake/ stores and hyperglycaemia, and to assess the joint effects on anaemia.

Design: We followed 1056 healthy adults aged 20 years and older from 2002 to 2007. Body Fe stores were measured. Dietary data were collected using a $3 \mathrm{~d}$ food record and FFQ. Hyperglycaemia was defined as fasting plasma glucose $>5.6 \mathrm{mmol} / 1$.

Results: Of the participants, $28 \cdot 8 \%$ were anaemic at baseline. During the 5 years of follow-up, we documented 125 incident cases of hyperglycaemia, among them twenty-three were diabetic. Haem Fe intake was positively associated with the risk of hyperglycaemia in men and women: the OR (95\% CI) across increasing quartiles of haem Fe intake was $1 \cdot 00$ (referent), $1 \cdot 49(0 \cdot 74,3 \cdot 01), 2 \cdot 16(1 \cdot 06,4 \cdot 42)$ and $3 \cdot 48(1 \cdot 71,7 \cdot 11)$, respectively ( $P$ for trend $<0 \cdot 001)$. Comparing the fourth quartile of serum ferritin with the others, the age- and gender-adjusted OR $(95 \%$ CI) was $1 \cdot 54(1 \cdot 01,2 \cdot 34), P$ for trend $=0 \cdot 043$. The association between total $\mathrm{Fe}$ intake and the risk of hyperglycaemia was significant in men $(P$ for trend $=$ $0 \cdot 002$ ). Anaemia added additional risk of hyperglycaemia on haem Fe intake. Comparing extreme quartiles of haem Fe intake, the OR (95\% CI) was $5 \cdot 67$ (1.43, $22 \cdot 49)$ and $3 \cdot 44(1 \cdot 51,7 \cdot 85)$ for hyperglycaemia among anaemic and non-anaemic participants ( $P$ for trend $=0 \cdot 008$ and $0 \cdot 010$, respectively).

Conclusions: The present cohort study suggests that high haem Fe intake, anaemia and high ferritin are associated with an increased risk of hyperglycaemia in Chinese men and women. There was a joint effect between anaemia and haem Fe intake on the risk of hyperglycaemia.
\end{abstract}

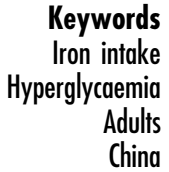

Like many in other countries of the world, diabetes has become a major public health concern in China. In 2002, it affected up to $13 \cdot 1 \%$ of urban inhabitants aged 60 years and older ${ }^{(1)}$. At the same time, the prevalence of anaemia in China is decreasing but remaining high $(15 \cdot 2 \%$ in $2002)^{(1)}$. Diet plays an important role in both diseases ${ }^{(2,3)}$. While Fe deficiency may lead to anaemia ${ }^{(3)}$, findings from many countries show that high Fe intake, especially haem Fe intake ${ }^{(4)}$, and high body Fe stores increase the risk of diabetes $^{(5-7)}$. Possible mechanisms for Fe increasing the risk of diabetes may include oxidative stress, inflammation, free fatty acid oxidation, insulin extraction and secretion $^{(6)}$. Anaemia is found to be negatively related to diabetes and gestational diabetes in some studies ${ }^{(8,9)}$, but anaemia is common among diabetes patients and increases the risk of morbidity and mortality ${ }^{(10)}$.

Fe-fortified soya sauce (using NaFeEDTA) is used in the prevention of anaemia in China. The objective of this intervention is to cover a population of 360 million (http://www.gainhealth.org/china-nafeedta-fortified-soysauce). We have previously shown in a cross-sectional study in south China that $\mathrm{Fe}$ intake, $\mathrm{Hb}$ and ferritin levels are associated with the risk of diabetes ${ }^{(8)}$. Similar results have been found in other studies from China ${ }^{(11)}$. Fe status is also related to gestational diabetes and insulin resistance in the Chinese population ${ }^{(9,12)}$. However, no cohort study on the association between $\mathrm{Fe}$ and diabetes in China is available. 
In order to prevent both anaemia and the diabetes epidemic simultaneously in countries like China, a clear understanding of the relationships between dietary $\mathrm{Fe} /$ body Fe stores and diabetes and anaemia is important. Using prospective data from the Jiangsu Nutrition Study (JIN), the objectives of the present paper are: (i) to prospectively examine for the first time whether dietary intake/ body stores of Fe is related to risk of hyperglycaemia in the Chinese population; and (ii) in light of the Fe supplement policy in China, to clarify the combined and stratified effects of Fe intake and anaemia on hyperglycaemia.

\section{Research design and methods}

\section{Sample}

The JIN is an ongoing cohort study investigating the association between nutrition and non-communicable chronic disease. It uses the sub-sample from Jiangsu Province of the Chinese National Nutrition and Health Survey in 2002 as baseline. The rural sample was selected from six counties (Jiangyin, Taichang, Shuining, Jurong, Sihong and Haimen). From each of the six counties, three smaller towns were randomly selected. The urban sample was selected from the capital cities of the two prefectures, Nanjing and Xuzhou; and from each capital city three streets were randomly selected. The six counties and the two prefectures represented a geographically and economically diverse population. In each town/street, two villages/neighbourhoods were randomly selected, and ninety households were further selected randomly from each village/neighbourhood. All the members in the households were invited to take part in the study. In addition, one-third of the households were interviewed for dietary intake, and all family members aged 3 years and older from these households were invited to give fasting blood samples. Written consents were obtained from all participants. The study was approved by Jiangsu Provincial Center for Disease Control and Prevention.

In 2007, a 5-year follow-up survey was done. To increase the number of rural participants, two counties Dafeng (from the north) and Changshu (from the south) were included. All participants aged 3 years and older were invited to give fasting flood samples. In total, 10475 fasting blood samples were collected in the 2007 survey.

In 2002, 2849 adults aged 20 years and above had fasting blood samples measured for glucose and $\mathrm{Hb}$, and dietary information was obtained. In 2007, only 1682 participants could be identified, 1492 of them participated in the study, and 1175 had fasting blood samples measured. The rest of the participants had either migrated to other cities for temporary work or moved out of the original streets to other streets within the urban area.

For the current analysis, we included study participants with fasting plasma glucose (FPG) $<5.6 \mathrm{mmol} / 1$ in 2002 and without known diabetes. The final sample in the study consisted of 445 men and 611 women.
Compared with the retained participants, those lost to follow-up were generally younger $(45 \cdot 5 v \cdot 49 \cdot 3$ years $)$. No differences in mean BMI, waist circumference, glucose and energy intake were found. However, those lost to follow-up had higher intake of total Fe $(26 \cdot 2 v .25 \cdot 9 \mathrm{mg} / \mathrm{d})$ and lower haem Fe intake $(2 \cdot 1 v .2 \cdot 5 \mathrm{mg} / \mathrm{d})$.

Participants were interviewed in their homes by trained health workers using a standard questionnaire.

\section{Measurement of $\mathrm{Hb}$, serum ferritin and fasting plasma glucose}

An overnight fasting blood sample was collected from all study participants. The blood samples were analysed for plasma glucose and $\mathrm{Hb}$ in local Centers for Disease Control and Prevention. FPG was measured using an enzymatic (hexokinase) colorimetric test. Hb was measured by the cyanmethaemoglobin method ${ }^{(13)}$. Serum ferritin was analysed in a laboratory in the National Center for Disease Control and Prevention in Beijing using a commercially available RIA kit (Beijing North Institute of Biological Technology, Beijing, China). Serum ferritin was recoded into gender-specific quartiles in the data analysis. We defined diabetes as FPG $>7 \cdot 0 \mathrm{mmol} / \mathrm{l}$, hyperglycaemia as $\mathrm{FPG}>5.6 \mathrm{mmol} / \mathrm{l}^{(14)}$. Anaemia was defined as $\mathrm{Hb}$ below $13 \mathrm{~g} / \mathrm{dl}$ for men and $12 \mathrm{~g} / \mathrm{dl}$ for women $^{(15)}$. Fe-deficiency anaemia was defined as the presence of both anaemia and serum ferritin $<15 \mu \mathrm{g} / \mathrm{l}^{(3)}$.

\section{Dietary measurements}

Nutrient intakes were measured by $3 \mathrm{~d}$ weighed food records. Participants were instructed to undertake this for three consecutive days including one weekend day. The food consumption data were analysed using the Chinese Food Composition Table ${ }^{(16)}$. Fe intake (total, haem, and non-haem Fe) was recoded into quartiles in the analysis.

Dietary patterns were identified by factor analysis based on food intake assessed by FFQ, using standard principal component analysis, which has been described elsewhere ${ }^{(17)}$. In short, four food patterns were obtained. Factor 1 ('macho') was characterized by various kinds of animal foods and alcohol, i.e. foods commonly taken by men. The 'traditional' pattern (factor 2) loaded heavily on rice and fresh vegetables, and inversely on wheat flour. Factor 3 ('sweet tooth') contained cake, milk, yoghurt and drinks. Factor 4 ('vegetable-rich' pattern) included whole grains, fruits, root vegetables, fresh and pickled vegetables, milk, eggs and fish. The four factors explained $28.5 \%$ of the variance in intake.

\section{Assessment of covariates}

Cigarette smoking was assessed by asking about frequency of daily cigarette smoking. Alcohol consumption was assessed by asking the frequency and amount of alcohol/wine intake.

Assessment of sedentary activity was asked by the following question: "How many hours a day do you spend on 
reading, watching television and playing video games?' Questions were asked on active commuting, such as walking or bicycling for different purposes. The information on physical activity was collected using a validated physical activity questionnaire covering a time period of 1 year ${ }^{(18)}$.

At each study site, health workers measured height, weight and blood pressure according to standard protocols. Blood pressure was measured twice by mercury sphygmomanometer on the right upper arm of the subject, who was seated for $5 \mathrm{~min}$ before the measurement. The mean of these two measurements was used in the analyses. Hypertension was defined as systolic blood pressure above $140 \mathrm{mmHg}$ and/or diastolic blood pressure above $90 \mathrm{mmHg}$, or using antihypertensive drugs. Height was measured without shoes and weight was measured with light clothing. BMI was calculated as weight in kilograms divided by the square of the height in metres. Waist circumference was measured midway between the inferior margin of the last rib and the iliac crest in a horizontal plane.

Household income was assessed by questions on family income and number of persons in the household. Three income groups were constructed: 'low' (<1999 Yuan/ person), 'medium' (2000-4999 Yuan/person) and 'high' (>5000 Yuan/person). Education was recoded into three categories based on six categories of education levels in the questionnaire: 'low' (illiteracy, primary school), 'medium' (junior middle school) and 'high' (high middle school or higher). Occupation was recoded into labour or non-labour based on a question with twelve occupational categories. Family history of diabetes was defined as the presence of known family members with type 2 diabetes in any of three generations (siblings, parents or grandparents). Known diabetes was based on self-reported hospital diagnosis of diabetes, as diabetes diagnosed by family practitioners was not common in the region.

\section{Statistical analyses}

The $\chi^{2}$ test was used to compare differences in categorical variables, and ANOVA was used to compare differences in continuous variables between groups. The association between Fe intake and the risk of hyperglycaemia was analysed using logistic regression models adjusting for multiple covariates. The logistic model controlled for age (continuous), sex, BMI, energy intake, education, smoking, sedentary activity, family history of diabetes (yes/no), ferritin, $\mathrm{Hb}$ and food patterns. Interaction was tested using the likelihood ratio method. Statistical significance was considered when $P<0.05$ (two-sided). All analyses were performed using the STATA statistical software package version 10 (Stata Corp, College Station, TX, USA).

\section{Results}

The mean intake of Fe was $25.0 \mathrm{mg} / \mathrm{d}$. Most of the total Fe intake $(90 \cdot 3 \%)$ was from non-haem Fe. Fe intake was positively associated with energy intake (Table 1). Compared with the lowest quartile, participants in the highest quartile of haem Fe intake consumed more energy, more energy from fat and had higher serum ferritin levels, but were more likely to be anaemic. They were also more likely to be daily drinkers and smokers. In total, $28 \cdot 8 \%$ of the participants had anaemia at baseline. However, the prevalence of anaemia with serum ferritin $<15 \mu \mathrm{g} / \mathrm{l}$ was only $3.9 \%$. During the 5-year follow-up of 1056 adults (445 men, 611 women), we identified 125 cases of hyperglycaemia, among them twenty-three were cases of diabetes. Eight participants started taking diabetic medication during the follow-up.

Intake of haem Fe was positively associated with the risk of hyperglycaemia (Table 2). After adjusting for known type 2 diabetes risk factors, the OR (95\% CI) of hyperglycaemia across quartiles of haem $\mathrm{Fe}$ intake was $1 \cdot 00$ (referent), $1 \cdot 49(0 \cdot 74,3 \cdot 01), 2 \cdot 16(1 \cdot 06,4 \cdot 42)$ and $3 \cdot 48(1 \cdot 71,7 \cdot 11)$, respectively ( $P$ for trend $<0 \cdot 001)$. Excluding those who took diabetic medication during follow-up did not change the association. When diabetes was used as outcome, the positive association between haem Fe intake and risk of diabetes was statically significant although the confidence interval was wide due to the small number of cases ( $n$ 23). The OR (95\% CI) of diabetes across quartiles of haem $\mathrm{Fe}$ intake was 1.00 (referent), $5.96(0.92,38 \cdot 46), 16.49(2.49,109 \cdot 26)$ and $9 \cdot 84(1 \cdot 41,68 \cdot 75)$, respectively $(P$ for trend $=0.033)$. When we used FPG $>6.1 \mathrm{mmol} / \mathrm{l}$ or diabetes as outcome, the OR (95\% CI) across quartiles of haem $\mathrm{Fe}$ intake was $1 \cdot 00$ (referent), $2 \cdot 36(0 \cdot 93,5 \cdot 99), 3 \cdot 51$, (1.32, 9.34) and $5 \cdot 18(1 \cdot 94,13 \cdot 80)$, respectively ( $P$ for trend $<0 \cdot 001)$.

Red meat intake was positively associated with the risk of hyperglycaemia. In multivariate analysis (adjusted for variables cited in Table 2, model 3), the OR (95\% CI) for hyperglycaemia across quartiles of red meat intake was 1.00 (referent), $0.73(0.42,1.27), 1 \cdot 24(0.73,2 \cdot 12)$ and $1.50(0.85,2 \cdot 66)$, respectively $(P$ for trend $=0.04)$. However, the association became non-significant after adjustment for haem Fe intake. The association between haem Fe intake and hyperglycaemia was independent of red meat intake (data not shown). Adding baseline glucose (continuous) into the multivariate analysis did not change the association between Fe intake and hyperglycaemia (data not shown).

Total intake of $\mathrm{Fe}$ was positively associated with hyperglycaemia in men but not in women. The OR (95\% CI) of hyperglycaemia across total $\mathrm{Fe}$ intake quartiles in multivariate analysis (adjusted for variables cited in Table 2, model 3) was $1 \cdot 00$ (referent), $1 \cdot 47(0 \cdot 57,3 \cdot 78)$, $1.38(0.49,3 \cdot 86)$ and $3.51(1 \cdot 10,11 \cdot 24)$, respectively ( $P$ for trend $=0.043$ ) in men; and 1.00 (referent), 0.86 $(0 \cdot 39,1 \cdot 87), 1 \cdot 41(0 \cdot 61,3 \cdot 29)$ and $1 \cdot 22(0 \cdot 41,3 \cdot 66)$, respectively ( $P$ for trend $=0 \cdot 518$ ) in women. Non-haem Fe intake was not associated with risk of hyperglycaemia. 
Table 1 Sample characteristics according to extreme quartiles (Q1 lowest and Q4 highest) of dietary iron intake at baseline in the Jiangsu Nutrition Study, 2002

\begin{tabular}{|c|c|c|c|c|c|c|c|c|c|c|c|c|}
\hline & \multicolumn{4}{|c|}{ Total Fe } & \multicolumn{4}{|c|}{ Haem Fe } & \multicolumn{4}{|c|}{ Non-haem Fe } \\
\hline & \multicolumn{2}{|c|}{ Q1 } & \multicolumn{2}{|c|}{ Q4 } & \multicolumn{2}{|c|}{ Q1 } & \multicolumn{2}{|c|}{ Q4 } & \multicolumn{2}{|l|}{ Q1 } & \multicolumn{2}{|c|}{ Q4 } \\
\hline & Mean or $\%$ & SD & Mean or \% & SD & Mean or \% & SD & Mean or $\%$ & SD & Mean or $\%$ & SD & Mean or $\%$ & SD \\
\hline Age (years) & $50 \cdot 5$ & $14 \cdot 8$ & $47 \cdot 0$ & $12 \cdot 2$ & $51 \cdot 0$ & $13 \cdot 5$ & $46 \cdot 7$ & $12 \cdot 5$ & $49 \cdot 8$ & $14 \cdot 6$ & $48 \cdot 1$ & $12 \cdot 5$ \\
\hline BMI $\left(\mathrm{kg} / \mathrm{m}^{2}\right)$ & $23 \cdot 1$ & $3 \cdot 6$ & $23 \cdot 4$ & $3 \cdot 0$ & $23 \cdot 8$ & 3.5 & $23 \cdot 3$ & $3 \cdot 0$ & $23 \cdot 1$ & 3.6 & $23 \cdot 3$ & $3 \cdot 1$ \\
\hline \multicolumn{13}{|l|}{ Nutrient/food intake } \\
\hline Energy $(\mathrm{kJ} / \mathrm{d})$ & 7522 & 1595 & 12126 & 2704 & 9573 & 3095 & 10343 & 2478 & 7593 & 1637 & 12310 & 2721 \\
\hline Fat (\% of energy) & $32 \cdot 0$ & $8 \cdot 5$ & $29 \cdot 8$ & $9 \cdot 8$ & $24 \cdot 2$ & $7 \cdot 7$ & $35 \cdot 3$ & $8 \cdot 1$ & $33 \cdot 1$ & $8 \cdot 1$ & $28 \cdot 7$ & $9 \cdot 5$ \\
\hline Protein (\% of energy) & $13 \cdot 2$ & $2 \cdot 9$ & $13 \cdot 5$ & $2 \cdot 7$ & $11 \cdot 8$ & $2 \cdot 2$ & $15 \cdot 1$ & $2 \cdot 9$ & $13 \cdot 8$ & $3 \cdot 1$ & $13 \cdot 1$ & $2 \cdot 7$ \\
\hline Carbohydrate (\% of energy) & $54 \cdot 8$ & $9 \cdot 1$ & $56 \cdot 7$ & $10 \cdot 7$ & $64 \cdot 0$ & $7 \cdot 8$ & $49 \cdot 6$ & $8 \cdot 8$ & $53 \cdot 1$ & $8 \cdot 8$ & $58 \cdot 2$ & $10 \cdot 4$ \\
\hline Red meat $(\mathrm{g} / \mathrm{d})$ & $57 \cdot 7$ & $50 \cdot 3$ & $68 \cdot 8$ & $73 \cdot 1$ & $7 \cdot 6$ & $13 \cdot 6$ & 131.9 & $69 \cdot 5$ & $75 \cdot 6$ & $62 \cdot 4$ & $54 \cdot \overline{8}$ & $63 \cdot 9$ \\
\hline Family history of diabetes (\%) & $2 \cdot 3$ & & 1.5 & & $1 \cdot 1$ & & $3 \cdot 4$ & & $2 \cdot 3$ & & $1 \cdot 1$ & \\
\hline Log ferritin $(\mu \mathrm{g} / \mathrm{l})$ & $4 \cdot 1$ & 0.9 & $4 \cdot 2$ & $1 \cdot 0$ & 3.9 & $1 \cdot 1$ & $4 \cdot 3$ & $1 \cdot 0$ & $4 \cdot 1$ & 0.9 & $4 \cdot 1$ & $1 \cdot 0$ \\
\hline $\mathrm{Hb}(\mathrm{g} / \mathrm{dl})$ & $12 \cdot 8$ & $1 \cdot 7$ & $13 \cdot 7$ & $1 \cdot 7$ & $13 \cdot 4$ & $1 \cdot 7$ & $13 \cdot 2$ & $1 \cdot 7$ & $12 \cdot 7$ & $1 \cdot 7$ & $13 \cdot 6$ & $1 \cdot 8$ \\
\hline Anaemia (\%) & $36 \cdot 0$ & & $22 \cdot 4$ & & $19 \cdot 3$ & & $34 \cdot 1$ & & $40 \cdot 2$ & & $22 \cdot 4$ & \\
\hline Hypertension (\%) & $26 \cdot 9$ & & $26 \cdot 9$ & & $37 \cdot 1$ & & $26 \cdot 9$ & & $28 \cdot 0$ & & $28 \cdot 4$ & \\
\hline \multicolumn{13}{|l|}{ Smoking (\%) } \\
\hline Never & $86 \cdot 0$ & & $63 \cdot 6$ & & $77 \cdot 3$ & & $61 \cdot 4$ & & $84 \cdot 1$ & & $65 \cdot 5$ & \\
\hline $1-19$ cigarettes/d & $9 \cdot 1$ & & $14 \cdot 8$ & & $12 \cdot 9$ & & $14 \cdot 8$ & & $9 \cdot 5$ & & $14 \cdot 8$ & \\
\hline$\geq 20$ cigarettes $/ \mathrm{d}$ & 4.9 & & $21 \cdot 6$ & & $9 \cdot 8$ & & $23 \cdot 8$ & & $6 \cdot 4$ & & $19 \cdot 7$ & \\
\hline \multicolumn{13}{|l|}{ Drinking (\%) } \\
\hline 0 times/week & $87 \cdot 1$ & & $63 \cdot 9$ & & $80 \cdot 2$ & & $66 \cdot 3$ & & $86 \cdot 7$ & & $65 \cdot 4$ & \\
\hline $1-2$ times/week & $4 \cdot 6$ & & $7 \cdot 6$ & & 6.5 & & $5 \cdot 7$ & & $4 \cdot 2$ & & $7 \cdot 2$ & \\
\hline 3-4 times/week & $2 \cdot 7$ & & $5 \cdot 3$ & & $2 \cdot 3$ & & $6 \cdot 4$ & & $2 \cdot 7$ & & 4.9 & \\
\hline Daily & $5 \cdot 7$ & & $23 \cdot 2$ & & $11 \cdot 0$ & & $21 \cdot 6$ & & $6 \cdot 4$ & & $22 \cdot 4$ & \\
\hline \multicolumn{13}{|l|}{ Education (\%) } \\
\hline Primary & $57 \cdot 2$ & & $51 \cdot 1$ & & $70 \cdot 1$ & & $40 \cdot 9$ & & $55 \cdot 3$ & & $57 \cdot 6$ & \\
\hline Junior school & $31 \cdot 8$ & & $39 \cdot 0$ & & $24 \cdot 6$ & & $43 \cdot 9$ & & $34 \cdot 1$ & & $33 \cdot 7$ & \\
\hline High school/university & $11 \cdot 0$ & & $9 \cdot 9$ & & $5 \cdot 3$ & & $15 \cdot 2$ & & $10 \cdot 6$ & & $8 \cdot 7$ & \\
\hline \multicolumn{13}{|l|}{ Active commuting (\%) } \\
\hline None & $54 \cdot 2$ & & $29 \cdot 2$ & & $29 \cdot 6$ & & $43 \cdot 2$ & & $56 \cdot 1$ & & $26 \cdot 5$ & \\
\hline $1-30 \mathrm{~min} / \mathrm{d}$ & $41 \cdot \overline{7}$ & & $50 \cdot 0$ & & $48 \cdot 1$ & & $51 \cdot \overline{5}$ & & $40 \cdot 9$ & & $50 \cdot 0$ & \\
\hline$\geq 30 \mathrm{~min} / \mathrm{d}$ & $4 \cdot 2$ & & $20 \cdot 8$ & & $22 \cdot 3$ & & $5 \cdot 3$ & & $3 \cdot 0$ & & 23.5 & \\
\hline \multicolumn{13}{|l|}{ Leisure-time physical activity (\%) } \\
\hline None & $92 \cdot 0$ & & $93 \cdot 2$ & & $94 \cdot 3$ & & $91 \cdot 3$ & & $91 \cdot 7$ & & $93 \cdot 9$ & \\
\hline $1-30 \mathrm{~min} / \mathrm{d}$ & 3.4 & & $4 \cdot 2$ & & 3.8 & & $3 \cdot 8$ & & 3.0 & & $3 \cdot 8$ & \\
\hline$\geq 30 \mathrm{~min} / \mathrm{d}$ & $4 \cdot 6$ & & $2 \cdot 7$ & & 1.9 & & 4.9 & & $5 \cdot 3$ & & $2 \cdot 3$ & \\
\hline \multicolumn{13}{|l|}{ Sedentary activity (\%) } \\
\hline$<1 \mathrm{~h} / \mathrm{d}$ & $16 \cdot 3$ & & $20 \cdot 1$ & & $28 \cdot 0$ & & $9 \cdot 5$ & & $15 \cdot 9$ & & $21 \cdot 2$ & \\
\hline $1-2 \mathrm{~h} / \mathrm{d}$ & $28 \cdot 8$ & & $31 \cdot 8$ & & $40 \cdot 9$ & & 26.5 & & $25 \cdot 0$ & & $35 \cdot 6$ & \\
\hline $2-3 \mathrm{~h} / \mathrm{d}$ & $44 \cdot 7$ & & $38 \cdot 3$ & & $27 \cdot 3$ & & $47 \cdot 4$ & & $48 \cdot 1$ & & $37 \cdot 1$ & \\
\hline$\geq 3 \mathrm{~h} / \mathrm{d}$ & $10 \cdot 2$ & & $9 \cdot 9$ & & $3 \cdot 8$ & & $16 \cdot 7$ & & $11 \cdot 0$ & & $6 \cdot 1$ & \\
\hline
\end{tabular}


Table 2 Odds ratio and $95 \%$ confidence interval of hyperglycaemia according to quartile of total iron, haem iron and non-haem iron intake: Jiangsu Nutrition Study ( $n$ 1056)

\begin{tabular}{|c|c|c|c|c|c|c|c|c|c|}
\hline & \multicolumn{8}{|c|}{ Quartile of intake } & \multirow[b]{3}{*}{$P$ for trend } \\
\hline & \multicolumn{2}{|c|}{ Q1 } & \multicolumn{2}{|r|}{ Q2 } & \multicolumn{2}{|r|}{ Q3 } & \multicolumn{2}{|r|}{ Q4 } & \\
\hline & OR & $95 \% \mathrm{Cl}$ & OR & $95 \% \mathrm{Cl}$ & OR & $95 \% \mathrm{Cl}$ & OR & $95 \% \mathrm{Cl}$ & \\
\hline \multicolumn{10}{|l|}{ Total Fe } \\
\hline Median intake (mg/d) & \multirow{2}{*}{\multicolumn{2}{|c|}{$\begin{array}{c}16 \cdot 2 \\
32\end{array}$}} & \multirow{2}{*}{\multicolumn{2}{|c|}{$\begin{array}{c}20 \cdot 8 \\
29\end{array}$}} & \multicolumn{2}{|c|}{$25 \cdot 6$} & \multicolumn{2}{|r|}{34.9} & - \\
\hline No. of new cases & & & & & \multicolumn{2}{|c|}{31} & \multicolumn{2}{|r|}{33} & _- \\
\hline Model $1^{*}$ & 1.00 & ref & 0.87 & $0.51,1.50$ & 0.89 & $0.51,1.54$ & 0.98 & $0.57,1.69$ & 0.98 \\
\hline Model $2 \dagger$ & 1.00 & ref & 0.82 & $0.47,1.42$ & 0.87 & $0.50,1.51$ & 0.95 & $0.55,1.64$ & 0.933 \\
\hline Model 3‡ & 1.00 & ref & 0.97 & $0.54,1.75$ & $1 \cdot 23$ & $0.64,2 \cdot 37$ & $2 \cdot 09$ & $0.97,4.48$ & 0.06 \\
\hline Model $4 \S$ & 1.00 & ref & 0.95 & $0.52,1.73$ & $1 \cdot 26$ & $0.64,2 \cdot 48$ & 1.97 & $0.89,4.36$ & 0.082 \\
\hline Model 5II & 1.00 & ref & 0.93 & $0 \cdot 51,1 \cdot 70$ & $1 \cdot 18$ & $0.59,2 \cdot 35$ & $1 \cdot 81$ & $0.81,4.07$ & 0.142 \\
\hline \multicolumn{10}{|l|}{ Haem Fe } \\
\hline Median intake $(\mathrm{mg} / \mathrm{d})$ & \multirow{2}{*}{\multicolumn{2}{|c|}{$\begin{array}{c}0.1 \\
19\end{array}$}} & \multicolumn{2}{|r|}{$1 \cdot 2$} & \multicolumn{2}{|r|}{$2 \cdot 2$} & \multicolumn{2}{|r|}{$4 \cdot 4$} & - \\
\hline No. of new cases & & & \multicolumn{2}{|r|}{25} & \multicolumn{2}{|r|}{34} & \multicolumn{2}{|r|}{47} & - \\
\hline Model $1^{*}$ & 1.00 & ref & $1 \cdot 40$ & $0 \cdot 75,2 \cdot 62$ & $2 \cdot 02$ & $1 \cdot 11,3.66$ & $2 \cdot 99$ & $1 \cdot 68,5 \cdot 34$ & $<0.001$ \\
\hline Model $2 \dagger$ & 1.00 & ref & $1 \cdot 49$ & $0 \cdot 79,2 \cdot 81$ & $2 \cdot 21$ & $1 \cdot 20,4 \cdot 06$ & $3 \cdot 22$ & $1 \cdot 79,5 \cdot 81$ & $<0.001$ \\
\hline Model 3‡ & 1.00 & ref & $1 \cdot 49$ & $0 \cdot 74,3 \cdot 01$ & $2 \cdot 16$ & $1 \cdot 06,4 \cdot 42$ & $3 \cdot 48$ & $1 \cdot 71,7 \cdot 11$ & $<0.001$ \\
\hline Model $4 \S$ & 1.00 & ref & 1.58 & $0 \cdot 77,3 \cdot 24$ & $2 \cdot 32$ & $1 \cdot 11,4 \cdot 84$ & 3.65 & $1 \cdot 75,7 \cdot 63$ & $<0.001$ \\
\hline Model 5II & 1.00 & ref & 1.53 & $0.73,3.21$ & $2 \cdot 16$ & $1.02,4.58$ & $3 \cdot 23$ & $1.51,6.94$ & 0.001 \\
\hline \multicolumn{10}{|l|}{ Non-haem Fe } \\
\hline Median intake $(\mathrm{mg} / \mathrm{d})$ & \multirow{2}{*}{\multicolumn{2}{|c|}{$\begin{array}{c}14 \cdot 4 \\
36\end{array}$}} & \multirow{2}{*}{\multicolumn{2}{|c|}{$\begin{array}{c}18 \cdot 7 \\
34\end{array}$}} & \multirow{2}{*}{\multicolumn{2}{|c|}{$\begin{array}{c}22 \cdot 5 \\
28\end{array}$}} & & $32 \cdot 3$ & - \\
\hline No. of new cases & & & & & & & & 27 & - \\
\hline Model $1^{*}$ & 1.00 & ref & 0.90 & $0.54,1.50$ & 0.68 & $0 \cdot 39,1 \cdot 16$ & 0.65 & $0.38,1 \cdot 13$ & 0.077 \\
\hline Model 2† & 1.00 & ref & 0.89 & $0.53,1.50$ & 0.63 & $0.36,1.09$ & 0.64 & $0.37,1 \cdot 11$ & 0.06 \\
\hline Model 3ł & 1.00 & ref & 0.95 & $0.54,1.67$ & 0.72 & $0.38,1.39$ & 1.02 & $0.44,2 \cdot 36$ & 0.694 \\
\hline Model $4 \S$ & 1.00 & ref & 0.94 & $0.53,1.67$ & 0.73 & $0.38,1.43$ & 0.97 & $0.40,2.32$ & 0.662 \\
\hline Model 5II & 1.00 & ref & 0.90 & $0.50,1.62$ & 0.76 & $0.38,1.51$ & 0.95 & $0 \cdot 39,2 \cdot 31$ & 0.710 \\
\hline & & & & Ferritin & $1-3 v$ & & & & \\
\hline Median level $(\mu \mathrm{g} / \mathrm{l})$ & & & - & & - & & & $181 \cdot 2$ & \\
\hline No. of new cases & & & _- & & _- & & & 40 & \\
\hline Model $1^{*}$ & 1.00 & ref & - & & - & & 1.54 & $1 \cdot 01,2 \cdot 34$ & 0.043 \\
\hline Model 2t & 1.00 & ref & - & & - & & $1 \cdot 43$ & $0.94,2 \cdot 19$ & 0.096 \\
\hline Model 3ł & 1.00 & ref & - & & - & & 1.34 & $0 \cdot 86,2 \cdot 10$ & 0.198 \\
\hline
\end{tabular}

ref, referent category.

${ }^{*}$ Model 1 adjusted for age and gender.

†Model 2 adjusted for age, gender and BMI.

łModel 3 adjusted for age, gender, BMI (continuous); central obesity (waist circumference: men $\geq 90 \mathrm{~cm}$, women $\geq 80 \mathrm{~cm}$ ); hypertension; family history of diabetes (yes/no); sedentary physical activity ( $<1,1-2,2-3, \geq 3 \mathrm{~h} / \mathrm{d}$ ); active commuting (no, 1-30, $\geq 30$ min/d); cigarette smoking (never,1-19, $\geq 20$ cigarettes/d); alcohol consumption (0, 1-2 times/week, 3-4 times/week, daily); intakes of total energy, fat, fibre and Mg; education, income (low, medium, high), job (manual, non-manual).

\$Model 4 adjusted for variables in Model 3 and quartiles of ferritin and $\mathrm{Hb}$.

IIModel 5 adjusted for variables in Model 4 and four food pattern scores (continuous).

ๆTen subjects without serum ferritin measurement at baseline, two of them developed hyperglycaemia. The range of ferritin in Q4 was $96 \cdot 1-485 \cdot 3 \mu \mathrm{g} / \mathrm{l}$.

Stratified analysis showed that the association between haem Fe intake and hyperglycaemia was not modified by gender, smoking, drinking, BMI or food pattern (Table 3). The association was stronger among men, smokers, drinkers and those being anaemic. However, the multiplicative interactions were not statistically significant.

Adjusting for age and gender, persons with high serum ferritin (fourth quartile) had a higher risk of hyperglycaemia: OR $(95 \% \mathrm{CI})$ of $1.54(1 \cdot 01,2 \cdot 34), P$ for trend $=$ $0 \cdot 043$. This association became marginally significant in multivariate analysis. When serum ferritin was low (first quartile), anaemic participants had an OR (95\% CI) of $2 \cdot 48(1 \cdot 06,5 \cdot 80)$ for hyperglycaemia compared with nonanaemic participants $(P=0 \cdot 040$; data not shown). We also found a significant positive association between anaemia and the risk of hyperglycaemia. In multivariate analysis, comparing the anaemic with non-anaemic, the OR (95\% CI) for hyperglycaemia was $2.37(1.12,5.03)$ in men $(P=0.025), 1.41(0.75,2.63)$ in women $(P=0.287)$ and $1.52(1.01,2 \cdot 29)$ in both genders combined, $P=0.041$. The OR $(95 \%$ CI $)$ for hyperglycaemia became even stronger in the untreated anaemic persons when compared with non-anaemic: $1 \cdot 76(1 \cdot 14,2 \cdot 72), P=0 \cdot 015$.

We next tested the joint effects of anaemia and haem Fe intake on risk of hyperglycaemia (Fig. 1). Compared with the lowest intake of haem $\mathrm{Fe}$ and non-anaemic, those anaemic with the highest intake of haem Fe had an OR (95\% CI) of $5 \cdot 84(2 \cdot 40,14 \cdot 19), P<0 \cdot 001$. Similar joint effects were found between anaemia and total Fe intake on the risk of hyperglycaemia. For the non-anaemic, total Fe intake and especially haem Fe intake increased the risk. Fe in non-haem form did not increase the risk. 
Table 3 Stratified odds ratio and $95 \%$ confidence interval of hyperglycaemia according to quartile of iron intake*: Jiangsu Nutrition Study $(n$ 1056)

\begin{tabular}{|c|c|c|c|c|c|c|c|c|c|c|c|c|}
\hline & \multirow[b]{3}{*}{ No. of participants } & \multirow[b]{3}{*}{ No. of cases } & \multicolumn{8}{|c|}{ Quartile of intake } & \multirow[b]{3}{*}{$P$ for trend } & \multirow[b]{3}{*}{$P$ for interaction } \\
\hline & & & \multicolumn{2}{|c|}{ Q1 } & \multicolumn{2}{|r|}{ Q2 } & \multicolumn{2}{|r|}{ Q3 } & \multicolumn{2}{|r|}{ Q4 } & & \\
\hline & & & OR & $95 \% \mathrm{Cl}$ & $\mathrm{OR}$ & $95 \% \mathrm{Cl}$ & OR & $95 \% \mathrm{Cl}$ & OR & $95 \% \mathrm{Cl}$ & & \\
\hline $\begin{array}{l}\text { Haem Fe intake } \\
\text { Men } \\
\text { Women }\end{array}$ & $\begin{array}{l}445 \\
611\end{array}$ & $\begin{array}{l}65 \\
60\end{array}$ & $\begin{array}{l}1.00 \\
1.00\end{array}$ & $\begin{array}{l}\text { ref } \\
\text { ref }\end{array}$ & $\begin{array}{l}2.29 \\
1.14\end{array}$ & $\begin{array}{l}0 \cdot 67,7 \cdot 77 \\
0 \cdot 46,2 \cdot 85\end{array}$ & $\begin{array}{l}3 \cdot 28 \\
1 \cdot 70\end{array}$ & $\begin{array}{l}0.95,11 \cdot 34 \\
0 \cdot 65,4 \cdot 44\end{array}$ & $\begin{array}{l}6 \cdot 70 \\
2 \cdot 42\end{array}$ & $\begin{array}{l}1 \cdot 91,23 \cdot 46 \\
0.92,6 \cdot 34\end{array}$ & $\begin{array}{l}0.001 \\
0.040\end{array}$ & 0.462 \\
\hline $\begin{array}{l}\text { Smoker } \\
\text { Non-smoker }\end{array}$ & $\begin{array}{l}292 \\
764\end{array}$ & $\begin{array}{l}44 \\
81\end{array}$ & $\begin{array}{l}1.00 \\
1.00\end{array}$ & $\begin{array}{l}\text { ref } \\
\text { ref }\end{array}$ & $\begin{array}{l}2 \cdot 82 \\
1.35\end{array}$ & $\begin{array}{l}0.49,16 \cdot 44 \\
0.62,2 \cdot 96\end{array}$ & $\begin{array}{l}9 \cdot 29 \\
1.57\end{array}$ & $\begin{array}{l}1 \cdot 57,54 \cdot 98 \\
0.68,3 \cdot 61\end{array}$ & $\begin{array}{r}17 \cdot 45 \\
2 \cdot 38\end{array}$ & $\begin{array}{l}2 \cdot 88,105 \cdot 63 \\
1 \cdot 02,5 \cdot 57\end{array}$ & $\begin{array}{c}<0.001 \\
0.04\end{array}$ & $0 \cdot 150$ \\
\hline $\begin{array}{l}\text { Anaemic } \\
\text { Non-anaemic }\end{array}$ & $\begin{array}{l}304 \\
752\end{array}$ & $\begin{array}{l}48 \\
77\end{array}$ & $\begin{array}{l}1 \cdot 00 \\
1 \cdot 00\end{array}$ & $\begin{array}{l}\text { ref } \\
\text { ref }\end{array}$ & $\begin{array}{l}2 \cdot 19 \\
1.38\end{array}$ & $\begin{array}{l}0.54,8.92 \\
0.57,3.31\end{array}$ & $\begin{array}{l}2 \cdot 67 \\
2 \cdot 14\end{array}$ & $\begin{array}{l}0.66,10 \cdot 85 \\
0.87,5 \cdot 23\end{array}$ & $\begin{array}{l}5 \cdot 67 \\
2 \cdot 93\end{array}$ & $\begin{array}{l}1 \cdot 43,22 \cdot 49 \\
1 \cdot 17,7 \cdot 33\end{array}$ & $\begin{array}{l}0.008 \\
0.010\end{array}$ & 0.900 \\
\hline $\begin{array}{l}\mathrm{BMl}<24 \mathrm{~kg} / \mathrm{m}^{2} \\
\mathrm{BMl} \geq 24 \mathrm{~kg} / \mathrm{m}^{2}\end{array}$ & $\begin{array}{l}647 \\
408\end{array}$ & $\begin{array}{l}64 \\
61\end{array}$ & $\begin{array}{l}1.00 \\
1.00\end{array}$ & $\begin{array}{l}\text { ref } \\
\text { ref }\end{array}$ & $\begin{array}{l}1 \cdot 23 \\
2 \cdot 00\end{array}$ & $\begin{array}{l}0.47,3.20 \\
0.69,5 \cdot 81\end{array}$ & $\begin{array}{l}1.54 \\
2 \cdot 99\end{array}$ & $\begin{array}{l}0.58,4 \cdot 10 \\
0.99,9 \cdot 00\end{array}$ & $\begin{array}{l}3.73 \\
3.96\end{array}$ & $\begin{array}{l}1 \cdot 41,9 \cdot 89 \\
1 \cdot 29,12 \cdot 14\end{array}$ & $\begin{array}{l}0.003 \\
0.013\end{array}$ & 0.509 \\
\hline $\begin{array}{l}\text { Total Fe intake } \\
\text { Men } \\
\text { Women }\end{array}$ & $\begin{array}{l}445 \\
611\end{array}$ & $\begin{array}{l}65 \\
60\end{array}$ & $\begin{array}{l}1 \cdot 00 \\
1 \cdot 00\end{array}$ & $\begin{array}{l}\text { ref } \\
\text { ref }\end{array}$ & $\begin{array}{l}1.59 \\
0.72\end{array}$ & $\begin{array}{l}0 \cdot 60,4 \cdot 26 \\
0 \cdot 32,1 \cdot 60\end{array}$ & $\begin{array}{l}1.48 \\
1.31\end{array}$ & $\begin{array}{l}0 \cdot 51,4 \cdot 33 \\
0 \cdot 55,3 \cdot 13\end{array}$ & $\begin{array}{l}4.59 \\
1 \cdot 25\end{array}$ & $\begin{array}{l}1 \cdot 34,15 \cdot 72 \\
0 \cdot 41,3 \cdot 82\end{array}$ & $\begin{array}{l}0.002 \\
0.616\end{array}$ & 0.860 \\
\hline $\begin{array}{l}\text { Anaemic } \\
\text { Non-anaemic }\end{array}$ & $\begin{array}{l}304 \\
752\end{array}$ & $\begin{array}{l}48 \\
77\end{array}$ & $\begin{array}{l}1 \cdot 00 \\
1 \cdot 00\end{array}$ & $\begin{array}{l}\text { ref } \\
\text { ref }\end{array}$ & $\begin{array}{l}0.82 \\
1.15\end{array}$ & $\begin{array}{l}0 \cdot 29,2 \cdot 36 \\
0 \cdot 52,2 \cdot 54\end{array}$ & $\begin{array}{l}0 \cdot 60 \\
2 \cdot 03\end{array}$ & $\begin{array}{l}0 \cdot 16,2 \cdot 26 \\
0 \cdot 88,4 \cdot 69\end{array}$ & $\begin{array}{l}1 \cdot 06 \\
3 \cdot 80\end{array}$ & $\begin{array}{l}0 \cdot 25,4 \cdot 55 \\
1 \cdot 43,10 \cdot 10\end{array}$ & $\begin{array}{l}0.954 \\
0.005\end{array}$ & 0.760 \\
\hline $\begin{array}{l}\mathrm{BMl}<24 \mathrm{~kg} / \mathrm{m}^{2} \\
\mathrm{BMI} \geq 24 \mathrm{~kg} / \mathrm{m}^{2}\end{array}$ & $\begin{array}{l}647 \\
408\end{array}$ & $\begin{array}{l}64 \\
61\end{array}$ & $\begin{array}{l}1.00 \\
1.00\end{array}$ & $\begin{array}{l}\text { ref } \\
\text { ref }\end{array}$ & $\begin{array}{l}1.40 \\
0.74\end{array}$ & $\begin{array}{l}0.62,3 \cdot 16 \\
0.29,1.90\end{array}$ & $\begin{array}{l}1.35 \\
1.36\end{array}$ & $\begin{array}{l}0.54,3.41 \\
0.48,3.84\end{array}$ & $\begin{array}{l}3 \cdot 21 \\
1 \cdot 99\end{array}$ & $\begin{array}{l}1 \cdot 10,9 \cdot 35 \\
0.58,6 \cdot 80\end{array}$ & $\begin{array}{l}0.059 \\
0.197\end{array}$ & 0.867 \\
\hline
\end{tabular}

ref, referent category.

*Adjusted for BMI (continuous); central obesity (waist circumference: men $\geq 90 \mathrm{~cm}$, women $\geq 80 \mathrm{~cm}$ ); hypertension; family history of diabetes (yes/no); sedentary physical activity ( $<1,1-2,2-3, \geq 3 \mathrm{~h} / \mathrm{d}$ ); active commuting (no, 1-30, $\geq 30 \mathrm{~min} / \mathrm{d}$ ); cigarette smoking (never, $1-19, \geq 20$ cigarettes/d); alcohol consumption ( $0,1-2$ times/week, $3-4$ times/week, daily); intakes of total energy, fat, fibre and Mg; education, income (low, medium, high), (the model. 
(a)

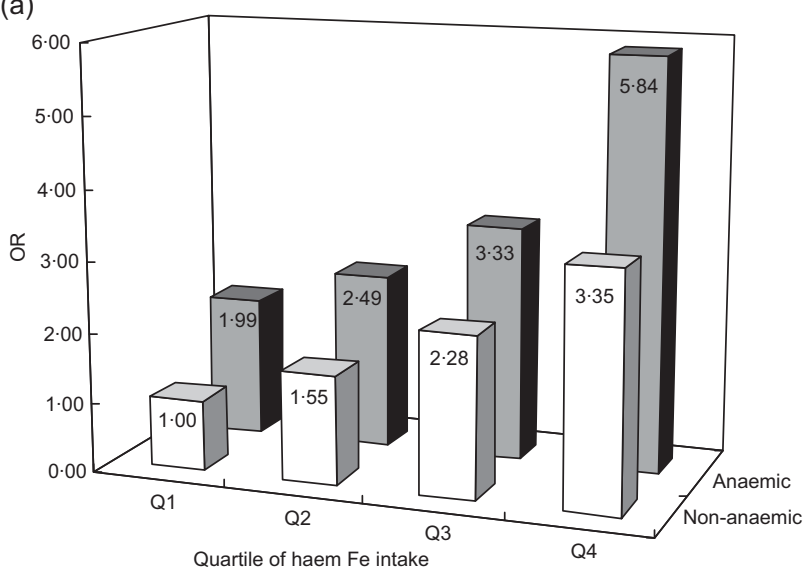

(b)

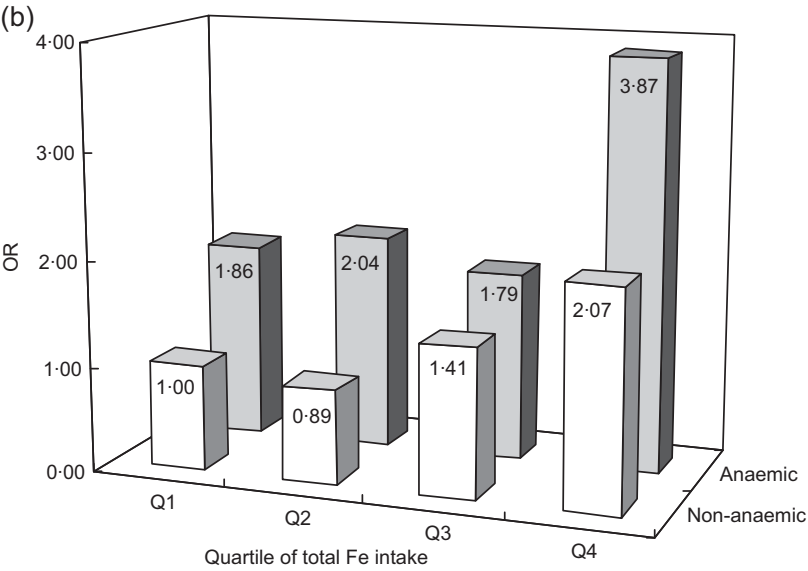

Fig. 1 Joint effects of anaemia and (a) haem iron intake and (b) total iron intake on risk of hyperglycaemia among Chinese adults (adjustment for variables cited in Table 2, model 3): Jiangsu Nutrition Study

\section{Discussion}

In the first prospective study on the association between Fe intake, anaemia and the risk of hyperglycaemia in a Chinese population, we found a positive association between the intake of haem $\mathrm{Fe}$ and hyperglycaemia. Intake of total Fe was positively associated with the risk of hyperglycaemia in men but not in women. A borderline significant positive association between high ferritin level and hyperglycaemia was found.

Our results on the association between haem Fe intake, Fe status and abnormal glucose level are consistent with findings from Western countries ${ }^{(7,19-22)}$. They also confirm the cross-sectional findings of the association between $\mathrm{Fe}$ intake and $\mathrm{Fe}$ level and diabetes in China ${ }^{(8,11)}$. However, the results are different from our previous cross-sectional findings ${ }^{(8,23)}$ which showed that total and non-haem Fe intakes were associated with increased risk of diabetes only in women. In the present study, haem Fe and total Fe intakes were associated with abnormal glucose in men, while in women only the relationship with haem Fe intake was significant. This discrepancy could be explained in the cross-sectional study by an inverse causal link, namely that a change in diet had occurred due to disease, especially among women. Alternatively, intake of haem $\mathrm{Fe}$ could be a marker for unknown factors in meat; however, adjusting for intake of red meat did not change the association between haem Fe and hyperglycaemia. Thus, the effect of haem $\mathrm{Fe}$ is independent of red meat intake.

A positive association between anaemia and risk of hyperglycaemia was found, in contrast to the observations in the previous cross-sectional study. This discrepancy may be due to the limitations of a cross-sectional study design (e.g. selection and survival bias) that more likely lead to a spurious relationship. Earlier findings revealed that the coexistence of anaemia and metabolic syndrome is about $3 \%$ in the study population, while only $7 \%$ had anaemia but without any component of the metabolic syndrome ${ }^{(24)}$. In addition, Helicobacter pylori infection is reported to affect more than $60 \%$ of this population ${ }^{(25)}$ and inflammation is found to increase the risk of hyperglycaemia $^{(26)}$. It is thus likely that anaemia caused by infection or inflammation in this population also could be associated with an increase in the risk of hyperglycaemia. A borderline significant association between serum ferritin, a marker of inflammation ${ }^{(27)}$, and hyperglycaemia supports this hypothesis. Additive interaction between Fe especially haem Fe and anaemia may be mediated by inflammation/infection because bacteria like $H$. pylori depend on Fe to survive. A positive association between haem Fe intake and anaemia in the sample may also be explained by infections like H. pylori. Further research on the effects of systemic inflammation on anaemia and hyperglycaemia is needed.

Our study has public health significance. In the present population, more than a quarter was anaemic. The intervention programme launched in Jiangsu Province in 2003 using Fe-fortified soya sauce was based on the high prevalence of anaemia that was found in the baseline survey of 2002. Research from the USA shows that Fe supplementation did not increase the risk of diabetes ${ }^{(19)}$ in men, but increased the risk of diabetes in women ${ }^{(20)}$. Because only few subjects in our cohort used Fe-fortified soya sauce, it is difficult to test whether Fe specifically from this supplement increased the risk of hyperglycaemia. However, our data clearly indicate that high $\mathrm{Fe}$ intake (total and haem $\mathrm{Fe}$ ) increases hyperglycaemia. In addition, anaemia increases the risk of hyperglycaemia, and shows additive effects with Fe intake. These results could challenge the anaemia intervention strategy of Fe supplementation. Recommendations for Fe supplementation in the prevention of anaemia in this population should therefore be cautiously re-examined if more studies confirm our findings. More research is needed before a public health recommendation can be made. If inflammation or infection (e.g. H. pylori) is one of the 
main causes of anaemia, controlling these conditions should be considered the major approach in the prevention of anaemia. Fe supplementation is at least not a risk-free policy for anaemic patients regarding the risk of hyperglycaemia and diabetes.

The limitation of the study is the high rate of those lost to follow-up due to migration and city construction. However, loss to follow-up may not influence the findings to a large extent since the exposure variables were measured at baseline. Owing to the small number of incident cases of diabetes, we were not able to use diabetes as the end point. Another limitation is that we do not have markers of inflammation or infection, such as C-reactive protein levels. Raised level of C-reactive protein was found to be related to increased risk of metabolic syndrome in another study from China ${ }^{(28)}$. As information on menopause was not available, we were not able to include this as a possible confounder in the analysis on women. The strength is that we used FPG, which is not usually measured in large cohort studies. Adding dietary pattern as a covariate strengthened the findings on the link between Fe and abnormal blood glucose. Furthermore, nutrient intake was assessed by a weighed food record.

In conclusion, intake of haem Fe was positively associated with risk of hyperglycaemia prospectively in both men and women in a Chinese population, independent of dietary pattern. Total $\mathrm{Fe}$ intake increased the risk of hyperglycaemia among men. Anaemia and high ferritin were related to a higher risk of hyperglycaemia. More research is needed to establish the causal links between Fe intake, anaemia and hyperglycaemia.

\section{Acknowledgements}

Sources of funding: The study was financed by Jiangsu Provincial Natural Science Foundation BK2008464 and Jiangsu Provincial Health Bureau. Declaration of competing interests: Nothing to declare. Author contributions: Z.S. had full access to all data in the study and takes responsibility for the integrity of the data and the accuracy of the data analysis. Z.S. devised the study concept; Z.S., M.Z., B.Y., Y.D. and Y.L. acquired the data; Z.S. and L.Q. conducted the statistical analysis; Z.S. drafted of the manuscript; M.Z., B.Y., L.Q., Y.D., Y.L. and G.H.-O. critically revised the manuscript for important intellectual content. Acknowledgements: The authors thank the participating Regional Centers for Disease Control and Prevention in Jiangsu Province, including the Nanjing, Xuzhou, Jiangyin, Taicang, Suining, Jurong, Sihong and Haimen Centers, for their support in the data collection.

\section{References}

1. Li L, Rao K, Kong L et al. (2005) A description on the Chinese national nutrition and health survey in 2002. Zhonghua Liu Xing Bing Xue Za Zbi 26, 474-484.
2. Bantle JP, Wylie-Rosett J, Albright AL et al. (2008) Nutrition recommendations and interventions for diabetes: a position statement of the American Diabetes Association. Diabetes Care 31, Suppl. 1, S61-S78.

3. Zimmermann MB \& Hurrell RF (2007) Nutritional iron deficiency. Lancet 370, 511-520.

4. Rajpathak S, Ma J, Manson J et al. (2006) Iron intake and the risk of type 2 diabetes in women: a prospective cohort study. Diabetes Care 29, 1370-1376.

5. Swaminathan S, Fonseca VA, Alam MG et al. (2007) The role of iron in diabetes and its complications. Diabetes Care 30, 1926-1933.

6. Rajpathak SN, Crandall JP, Wylie-Rosett J et al. (2009) The role of iron in type 2 diabetes in humans. Biochim Biophys Acta 1790, 671-681.

7. Jiang R, Manson JE, Meigs JB et al. (2004) Body iron stores in relation to risk of type 2 diabetes in apparently healthy women. JAMA 291, 711-717.

8. Shi Z, Hu X, Yuan B, Pan X et al. (2006) Association between serum ferritin, hemoglobin, iron intake, and diabetes in adults in Jiangsu, China. Diabetes Care 29, $1878-1883$.

9. Lao TT \& Ho LF (2004) Impact of iron deficiency anemia on prevalence of gestational diabetes mellitus. Diabetes Care 27, 650-656.

10. Thomas MC (2007) Anemia in diabetes: marker or mediator of microvascular disease? Nat Clin Pract Nephrol 3, 20-30.

11. Luan DC, Li H, Li SJ et al. (2008) Body iron stores and dietary iron intake in relation to diabetes in adults in North China. Diabetes Care 31, 285-286.

12. Sheu WH, Chen YT, Lee WJ et al. (2003) A relationship between serum ferritin and the insulin resistance syndrome is present in non-diabetic women but not in non-diabetic men. Clin Endocrinol (Oxf) 58, 380-385.

13. Dallman PR (1984) Diagnosis of anemia and iron deficiency: analytic and biological variations of laboratory tests. Am J Clin Nutr 39, 937-941.

14. Genuth S, Alberti KG, Bennett P et al. (2003) Follow-up report on the diagnosis of diabetes mellitus. Diabetes Care 26, 3160-3167.

15. World Health Organization (1968) Nutritional Anaemias. Report of a WHO Scientific Group. WHO Technical Report Series no. 405, pp. 5-37. Geneva: WHO.

16. Yang Y (2005) Chinese Food Composition Table 2004 Beijing: Peking University Medical Press.

17. Shi Z, Hu X, Yuan B et al. (2008) Vegetable rich food pattern is related to obesity in China. Int J Obes (Lond) $\mathbf{3 2}$, 975-984.

18. Ma G, Luan D, Liu A et al. (2007) The analysis and evaluation of a physical activity questionnaire of Chinese employed population. Ying Yang Xue Bao 29, 217-221.

19. Jiang R, Ma J, Ascherio A et al. (2004) Dietary iron intake and blood donations in relation to risk of type 2 diabetes in men: a prospective cohort study. Am J Clin Nutr 79, $70-75$.

20. Lee DH, Folsom AR \& Jacobs DR Jr (2004) Dietary iron intake and type 2 diabetes incidence in postmenopausal women: the Iowa Women's Health Study. Diabetologia 47, 185-194.

21. Song Y, Manson JE, Buring JE et al. (2004) A prospective study of red meat consumption and type 2 diabetes in middle-aged and elderly women: the Women's Health Study. Diabetes Care 27, 2108-2115.

22. Tuomainen TP, Nyyssonen K, Salonen R et al. (1997) Body iron stores are associated with serum insulin and blood glucose concentrations. Population study in 1,013 eastern Finnish men. Diabetes Care 20, 426-428.

23. Shi Z \& Pan X (2008) Body iron stores and dietary iron intake in relation to diabetes in adults in north China: response to Luan et al. Diabetes Care 31, e25. 
24. Shi Z, Hu X, Yuan B et al. (2008) Coexistence of anaemia and the metabolic syndrome in adults in Jiangsu, China. Asia Pac J Clin Nutr 17, 505-513.

25. Shi R, Xu S, Zhang $\mathrm{H}$ et al. (2008) Prevalence and risk factors for Helicobacter pylori infection in Chinese populations. Helicobacter 13, 157-165.

26. Schmidt MI, Duncan BB, Sharrett AR et al. (1999) Markers of inflammation and prediction of diabetes mellitus in adults (Atherosclerosis Risk in Communities study): a cohort study. Lancet 353, 1649-1652.

27. Baynes RD (1996) Assessment of iron status. Clin Biochem 29, 209-215.

28. Ye X, Yu Z, Li H et al. (2007) Distributions of C-reactive protein and its association with metabolic syndrome in middle-aged and older Chinese people. J Am Coll Cardiol 49, 1798-1805. 